\title{
SHORT AND PREDICTIVE ASSESSMENT BATTERY OF FITNESS-TO-DRIVE AFTER STROKE
}

\author{
Emmanuel Abiodun Akinwuntan \\ School of Allied Health Sciences \\ Medical College of Georgia \\ Augusta, GA, USA \\ E-mail: aakinwuntan@mcg.edu \\ Hannes Devos, Hilde Feys, Geert Verheyden, Willy De Weerdt \\ Department of Rehabilitation Sciences \\ Faculty of Kinesiology and Rehabilitation Sciences \\ Katholieke Universiteit \\ Leuven, Belgium \\ Guido Baten \\ CARA, Belgian Road Safety Institute \\ Brussels, Belgium \\ Carlotte Kiekens \\ Department of Physical Medicine and Rehabilitation \\ University Hospital \\ Pellenberg, Belgium
}

\begin{abstract}
Summary: The objective of this study was to confirm the accuracy of a previously identified short assessment battery to predict fitness-to-drive after stroke in a new cohort of stroke survivors. This was a prospective study that included 43 (39 males and 4 females) participants who performed the pre-driving assessment that included a standardized on-road test at the Belgian Road Safety Institute in Brussels, Belgium. Participants were on average six months post stroke, not severely physically disabled, possessed valid drivers' licenses and actively drove prior to stroke onset. Fitness-to-drive decisions made based on performance in 15 tests of a full scale assessment battery were predicted using only scores in three previously identified predictive tests. Performance in the three tests (figure of Rey, visual neglect (lateralized mean reaction time) and on-road test) was used to correctly predict $37(86 \%)$ of the 43 participants' driving fitness. The sensitivity and specificity of the predictions were $77 \%$ and $92 \%$ respectively. The outcome of this study shows that the short assessment battery is indeed a good predictor of fitness-to-drive in stroke survivors, especially those without severe deficits.
\end{abstract}

\section{INTRODUCTION}

Though mortality rate due to stroke in industrialized countries is decreasing (Sarti et al., 2000), stroke is still one of the major causes of death and disabilities around the world. The annual incidence of new or recurrent stroke in Belgium is approximately 185 per 100,000 population, of which about $47 \%$ die within the first 12 months of the incident (Devroey, Cateren, \& Buntinx, 2003). In most countries, about $50 \%$ of stroke survivors wish to resume driving (Logan, Dyas, \& Gladman, 2004). In Belgium, survivors must wait for a minimum period of six months after stroke before evaluation of their driving fitness at the Centre for Determination of Fitness to 
Drive and Car Adaptations (CARA), a unit of the Belgian Road Safety Institute in Brussels (Van Den Meerschaut, 1998). The driving fitness evaluation performed at CARA usually includes visual, neuropsychological and on-road tests. In an earlier study (Akinwuntan et al., 2006), a model containing three tests (figure of Rey, visual neglect (lateralized mean reaction time) and on-road test) was identified as the most predictive of the decision of driving fitness of stroke survivors. A discriminant equation based on the three tests was used to correctly predict $87 \%$ of participants' driving fitness. The sensitivity and specificity of the predictions were $79 \%$ and $94 \%$ respectively. The aim in this study was to confirm the accuracy of the three tests to predict the driving fitness of a new cohort of stroke survivors.

\section{METHODS}

Stroke survivors who performed the 15 tests of the full scale battery of driving fitness after stroke, which included a standardized on-road test, were eligible for inclusion in the study. In the one-year period of the study, 46 persons met this criterion. Two had a history of epileptic fit in the last six months and were excluded in accordance with Belgian law (Van Den Meerschaut, 1998). One had severe receptive aphasia and was also excluded because of the need to comprehend instructions and tasks of the tests. In all, 39 males and 4 females (age $=55 \pm 12$ years) were included in the study. Twenty-two (51\%) of the participants had a left-sided brain lesion, 19 (44\%) had a right-sided lesion, while two (5\%) had bilateral lesion. Twenty-two (51\%) and $21(49 \%)$ sustained ischemic and hemorrhagic strokes, respectively. Participants were all at least six months post stroke (Median $=9$ months, Interquartile range $=7-20$ months) and could independently perform most functional activities of daily living (Barthel Index $\geq 75 / 100$ ), including independent ambulation with or without assistive devices. The average years of experience and distance driven annually of participants before onset of stroke were $33 \pm 11$ years and $24,000 \pm 27,000 \mathrm{~km}$, respectively. Four $(9 \%)$ participants had a history of aphasia but could clearly understand instructions and effectively communicate with the assessors. Six (14\%) and two $(5 \%)$ participants had hemi- and quadrant anopia, respectively, but were all certified by an ophthalmologist to be fit to perform the pre-driving assessment. Those with corrective glasses or lenses performed all tests of the pre-driving assessment using the appropriate corrections. All participants possessed valid drivers' licenses and actively drove prior to stroke onset.

Based on performance in the 15 tests of the full scale assessment battery, a legal pass or fail decisions of participants' driving fitness were made by the CARA team of assessors. Using a discriminant equation reported in an earlier study, which includes performance in three of the 15 tests, the legal driving fitness decisions were also predicted.

\section{RESULTS}

Twenty-six participants passed and 17 failed the pre-driving assessment based on performance in all 15 tests of the pre-driving assessment. With the discriminant equation (see below) from the earlier study (Akinwuntan et al., 2006), 37 (86\% i.e., $(a+d / a+b+c+d) * 100)$ of the 43 participants were correctly predicted to either pass or fail the pre-driving assessment, as seen in Table 1 . The sensitivity $(\mathrm{a} / \mathrm{a}+\mathrm{c}) * 100)$ and specificity of the predictions $(\mathrm{d} / \mathrm{b}+\mathrm{d}) * 100)$ were $77 \%$ and $92 \%$, respectively. 
Table 1. Comparisons of participants' predicted performances based on the discriminant equation versus actual performances

\begin{tabular}{lcc}
\hline & Participants' actual pass/fail performance \\
\hline Predicted pass/fail & Fail, n (\%) & Pass, n (\%) \\
Fail, n (\%) & $\mathbf{a}=13(30)$ & $\mathbf{b}=2(5)$ \\
Pass, n (\%) & $\mathbf{c}=4(9)$ & $\mathbf{d}=24(56)$ \\
\hline
\end{tabular}

\section{Discriminant Equation}

Pass $=($ Figure of Rey*8.74 + Visual Neglect (lateralized mean reaction time $) * 0.06+$ Road test score*0.14) -174.90

Fail $=($ Figure of Rey*8.51 + Visual Neglect $($ lateralized mean reaction time $) * 0.07+$ Road test score*0.08) - 162.66

When the Pass score is higher than the Fail score, a pass prediction is made and vice versa.

\section{DISCUSSION}

The predictive accuracy $(86 \%)$ of the short battery of three tests in this study remains impressive as in the earlier study (Akinwuntan et al., 2006). The specificity $(92 \%)$ of its prediction was again better than the sensitivity, an indication that the short battery is more accurate in identifying participants who will pass the pre-driving assessment. However, our inclusion criteria (based predominantly on Belgian laws [Van Den Meerschaut, 1998]) make it difficult to generalize our findings to prediction of driving fitness in the general stroke population. The mandatory six months wait period and other processes involved in performing the pre-driving assessment after stroke in Belgium sort of systematically excludes survivors with severe post stroke deficits. Due to use of similar inclusion criteria in this study as in the earlier one, a comparison of the descriptive characteristics of the population in both studies was done. Our finding revealed nearly similar population in both studies, with the exception of a higher percentage of hemorrhagic strokes in this study and presence of moderate or mild aphasia in the earlier study. It is therefore not known if the accuracy of the short battery of three tests to predict driving fitness will be the same, worse or even better in a cohort of stroke survivors with severe deficits or less than six months after onset of stroke.

\section{CONCLUSION}

The findings in this study confirm that the short battery of three tests is a very good predictor of driving fitness of stroke survivors, especially in those without severe deficits who are at least six months post stroke onset.

\section{ACKNOWLEDGMENT}

The primary author expresses profound appreciation to his Belgian colleagues, particularly those at the CARA section of the Belgian Institute for Road Safety, Brussels, Belgium, for support 
during the conduction of this study. The primary author also acknowledges the Department of Physical Therapy at the Medical College of Georgia, Augusta, GA, for funding support to attend the conference.

\section{REFERENCES}

Akinwuntan, A.E., Feys, H., De Weerdt, W., Baten, G., Arno, P., Kiekens, C. (2006). Prediction of driving after stroke: a prospective study. Neurorehabiltation and Neural Repair, 20, 417423.

Devroey, D., Van Cateren, V., Buntinx, F. (2003). Registration of stroke through the Belgian sentinel network and factors influencing stroke mortality. Cerebrovascular Disease, 16(3), 272-279.

Logan, P.A., Dyas, J., Gladman, J.R.F. (2004). Using an interview study of transport use by people who have had a stroke to inform rehabilitation. Clinical Rehabilitation, 18, 703-708.

Sarti, C., Rastenyte, D., Cepaitis, Z., Tuomilehto, J. (2000). International Trends in Mortality From Stroke, 1968 to 1994. Stroke 2000, 31, 1588-1601.

Van Den Meerschaut C. (1998). Rijgeschiktheid. Belgium: Semper October 1998;226:1-35. 